\title{
75 años de la Facultad de Medicina de la Pontificia U niversidad Católica de Chile
}

\author{
G onzalo G rebe $B^{1}$, Jorge Dagnino $S^{2}$, Ignacio Sánchez $D^{3}$. \\ Seventy five years of the Medical \\ School of the Pontifical Catholic \\ University of Chile
}

Aiming to join academic excellence and an ethical and Christian approach to medical profession, the Medical School of the Pontifical Catholic University of Chile initiated its activities in 1930. Since then, the associated Health Care Network has incorporated all the technological breakthroughs in medicine and developed all the specialties. Undergraduate teaching is oriented to promote creativity and innovation. There is also a special concern about humanity of Medicine, throught the Program of Humanistic Medical Studies and the Bioethics Center. Post graduate education is also an important activity of the School, through specialty training, Master and Doctorate programs. Researchers have also obtained important grants and generated a great number of publications in high impact journals. Our University is defined as «complex», meaning that we must take important challenges, be creative and lead knowledge generation. We must also improve ourselves to serve in the best possible way our students and the Country. Paraphrasing the words of our founder, Monsignor Carlos Casanueva, we must train physicians that will serve our community not only with science but also with humanity (Rev Méd Chile 2005; 133: 1229-32).

(Key Words: Education, medical; Humanities; Schools, medical; Religion)

Recibido el 9 de agosto, 2005. Aceptado el 15 de agosto, 2005.

${ }^{1}$ Decano, Facultad de Medicina; ${ }^{2}$ Vice-Decano, Facultad de Medicina; ${ }^{3}$ Director, Escuela de Medicina, Pontificia Universidad Católica de Chile.

Casi asi 40 años después de la fundación de la Universidad Católica de Chile en 1888, y posterior a varios intentos fallidos que habían fracasado por problemas principalmente de tipo financiero, en junio de 1929, el Arzobispado de Santiago dictaba el Decreto en virtud del cual se «declaraba definitivamente constituida la Facultad de Medicina de la Universidad Católica de Chile, en donde se nombra a su primer decano, el Dr. Carlos Monckeberg y se autorizaba la construcción inmediata en el local actual de la Universidad del pabellón de Anatomía y demás trabajos necesarios en dicho local». En los artículos del Decreto se autorizaba al Rector para contratar \os profesores que sean necesarios en el país o en el extranjero...., y para continuar los estudios del Hospital y policlínico complementarios de esta Escuela». Pocos días antes de comenzar las clases, el diario El Mercurio saludaba a la nueva Facultad reconociendo que sin el sespíritu avizor, tesonero, incansable y lleno de ideales»del Rector Monseñor Carlos Casanueva, hubiera sido muy 
difícil coronar con éxito los largos años de esfuerzo para ponerla en marcha. El comentarista señalaba que era cierto - como lo decía el Rectorque para surgir había necesitado la voluntad de Dios», pero advertía que también lo era que para mantenerse digna de su ideal necesita y necesitaría también de la voluntad serena y elevada de los hombres\%, lo que se ha cumplido a cabalidad en todos estos años en que el proyecto ha sido posible gracias al esfuerzo y generosidad de muchas personas.

La Escuela de Medicina inició sus actividades académicas en 1930, con el fin de aunar la excelencia científica con un profundo sentido ético y cristiano de la profesión médica. En 1942, se inauguró el Hospital Clínico con los Servicios de Medicina y Cirugía General de Adultos, realizando la docencia de las otras especialidades principales en campos clínicos asociados, incorporó el Servicio de Obstetricia en 1960, la Unidad de Neonatología en 1977, el Centro de Diagnóstico en 1980 y el Servicio de Pediatría en 1987. En 1955, la Escuela de Medicina, al completar sus estudios de Pre Grado, obtuvo la autorización legal para conceder el grado de licenciado en Medicina, y en 1981, la autonomía para formular sus planes de estudios y otorgar el título profesional de Médico Cirujano ${ }^{3}$. Desde su fundación, el Hospital Clínico ha incorporado los adelantos científico-tecnológicos de la medicina, desarrollando todas las especialidades clínicas en sus aspectos diagnósticos y terapéuticos. En 1989 se crea el Centro de Investigaciones Médicas, a lo que se agrega el Centro de Cáncer, y un crecimiento muy importante de la Red de Salud tanto en el ámbito hospitalario, como en forma especial en el ambulatorio ${ }^{4}$.

Los miembros de la Escuela de Medicina proyectan su quehacer al ámbito profesional, participando activamente en las diversas sociedades científicas del país, en comités editoriales de revistas científicas, siendo actualmente doce profesores integrantes de la Academia de Medicina del Instituto de Chile. Académicos y profesionales egresados de la Escuela de Medicina, han desempeñado responsabilidades de gobierno y cargos de jerarquía académica en Facultades de Medicina en otras Universidades, en el país y en el extranjero. Cabe destacar que cuatro miembros de la Facultad han sido distinguidos con el Premio Nacional de Ciencias.

Este aniversario de 75 años de actividades académicas, encuentra a la Escuela de Medicina en un momento de consolidación y de implementación de un variado número de proyectos que se resumirán a continuación, enfocándolos en cada una de las áreas académicas que son propias del quehacer universitario. En Pre Grado, nuestra Escuela -que actualmente tiene 786 alumnosincorporó hace varios años la reforma curricular de la Universidad, que tiene como objetivo entregar una formación general más global y amplia, por lo que existe un número significativo de créditos de formación humanista, artística y filosófica que colaboran a una mejor formación de los alumnos. Nuestros esfuerzos están orientados a la creatividad e innovación en docencia, la que incluye el desarrollo de proyectos como el de incrementar la docencia ambulatoria, el empleo de la simulación en la docencia, pacientes entrenados, métodos de evaluación que incorporen estos conceptos (OSCE y otros), docencia interactiva y personalizada, con el objeto a volver a tener un contacto personal y tutorial con el alumno, que es la base de la enseñanza y educación médica ${ }^{5}$. Otra idea de especial preocupación ha sido el cultivo de las humanidades en relación con la Medicina, a través de iniciativas como el Programa de Estudios Médicos Humanísticos y el Centro de Bioética. La creación hace 5 años de un Diploma de Formación en Docencia Clínica, ha permitido capacitar a nuestros docentes en nuevas técnicas y métodos de enseñanza de adultos. Nuestra Escuela se sometió en 1997 a un proceso de acreditación de la Asociación de Escuelas de Medicina Norteamericanas (AAMC), quedando aprobada, y en cuyo informe las sugerencias de mejoría fueron muy importantes para nuestro desarrollo. Posteriormente, en el año 2003, la carrera fue acreditada por el máximo de 7 años por la Comisión Nacional de Acreditación, dependiente del Ministerio de Educación. Nuestro próximo desafío es repetir el proceso de acreditación norteamericana, luego de 8 años de evolución y crecimiento, lo que por cierto permitirá un vigoroso desarrollo en diferentes áreas. En el Examen Médico Nacional, la Escuela de Medicina UC repitió el primer lugar en el año 2004, liderando en todas las áreas de especialidades, lo que nos 
compromete a seguir creciendo y superándonos y a aportar médicos con una formación de excelencia al servicio del país.

En Post Grado, nuestra Escuela cuenta con 385 alumnos, en 21 programas de especialidad, 26 de sub-especialidad, Doctorado en Ciencias Médicas y Magísteres en Nutrición y Bioética ${ }^{5}$. La dedicación exclusiva, exigida a todos nuestros alumnos, ha sido una política orientada a lograr una formación de excelencia, sin distracciones. Nuestros proyectos principales incluyen el desarrollo de un Magíster en Ciencias Médicas (con cursos transversales y una Tesis), y el acreditar los programas bajo estándares internacionales que nos permitirán dar un paso real en la calidad de nuestra enseñanza, lo que ciertamente tendrá un impacto nacional. Se han incrementado los convenios internacionales a nivel de Pre Grado con las Universidades de Harvard, Clínica Mayo. En el caso de Post Grado y académicos jóvenes, especial mención merece Washington University en Saint Louis; y en el área de investigación hemos hecho contactos formales con las Universidades de Washington en Seattle, Paris VI, Lille y Heidelberg. El desarrollo y estímulo a la investigación ha sido una labor prioritaria: actualmente existen 29 Proyectos Fondecyt vigentes, junto a otras investigaciones internacionales colaborativas. Más de 50 académicos desarrollan labores preferentes de investigación ( $8 \%$ del total), con 162 publicaciones científicas en el año 2004 (90 de ellos en revistas nacionales y extranjeras indexadas por ISI). Cabe hacer notar que desde el año 1967, la Facultad de Ciencias Biológicas inicialmente «nstituto de Biología», reúne a las ciencias básicas biológicas de la Universidad, como una forma de concentrar la docencia en esta área que es transversal para muchas carreras, y para formar grupos de investigación concursable de gran productividad, que representen liderazgo a nivel nacional ${ }^{6}$. Nuestros planes en esta área son potenciar el número de investigadores, asegurar tiempo protegido para estas labores, aumentar el espacio físico e infraestructura de los laboratorios, y preocuparnos de la formación de nuevos investigadores profesionales a través de nuestro programa de Doctorado. En el tema de las publicaciones, se debe trabajar en el impacto o índice de citación, que es el elemento que definirá la calidad del aporte internacional.
El grupo total de docentes de la Escuela asciende a 612, de los cuales 380 tienen jomada completa; ellos se desempeñan en diferentes campos clínicos docentes hospitalarios y ambulatorios, de los cuales los más importantes son el campus central de Marcoleta (Hospital Clínico, Centro de Cáncer y Áreas ambulatorias de Especialidades), el Hospital de Urgencia Asistencia Pública en la docencia de Pre y Postgrado de Medicina Interna y el Hospital Sótero del Río, en el área Sur Oriente de Santiago, el que, bajo el marco del convenio docente de más de 40 años de historia, renovado recientemente por un período de 15 años, permite que nuestros alumnos tengan la posibilidad de trabajar en un Hospital público y, por otra parte, aporta al Hospital más de 100 docentes que desempeñan su labor docente-asistencial, y un aporte económico anual que permite desarrollar proyectos de infraestructura que son claves para el desarrollo hospitalario, con impacto en la docencia y en la atención de los enfermos.

Quisiéramos destacar algunas iniciativas de gran trascendencia en el desarrollo de la Escuela: el proyecto Ancora, de Medicina Familiar, que consiste en el funcionamiento de 5 consultorios de Medicina Familiar en las comunas de Puente Alto y la Pintana, para desarrollar el modelo de atención primaria con 20.000 personas en cada uno. El primer centro ya lleva más de un año de actividad, están en construcción los siguientes 2 centros, que han tenido un gran impacto en la docencia de Pre y Post Grado, de tipo transversal e interdisciplinario con otros profesionales como enfermeras, psicólogas y asistentes sociales. El proyecto de desarrollar Medicina Paliativa al interior de la Escuela requiere una mención especial, ya que consideramos que debemos tener una postura académica, humanitaria y católica con el paciente terminal. Es por esto que se ha desarrollado un ambicioso proyecto multidisciplinario, con la participación de diferentes departamentos clínicos, realización de cursos y presencia de profesores invitados, renovación de un convenio marco con la Clínica Familia por 10 años, como campo clínico, con el objeto de desarrollar labores docente-asistenciales y de investigación, que puedan impactar en la formación de nuestros alumnos de Pre y Post Grado, y que beneficien a los 
pacientes de escasos recursos que padecen enfermedades terminales, en que el dolor y sufrimiento son la característica principal. El trabajo conjunto con la Fundación Josefina Martínez y el Hospital CEDERI, de Enfermedades Respiratorias en Niños Crónicos, en que se está desarrollando en conjunto un ambicioso programa de ventilación no invasiva y una atención más completa a los niños con patología respiratoria de larga evolución, que están hospitalizados por largos períodos en diferentes hospitales del país; y por último, el convenio docente asistencial con el Hogar de Cristo, que nos acerca a la población más desposeída de nuestro país, y que incluye la atención ambulatoria, hospitalaria y de evaluación diagnóstica.

Quisiéramos expresar lo más importante dentro de nuestra Misión y Objetivos como Escuela de Medicina de una Universidad Católica, en un país en vías de desarrollo. Nuestra Universidad se ha definido como compleja, y esto requiere aceptar los desafíos de ser creativos, líderes en la generación de conocimiento y estar permanentemente superándonos para entregar lo mejor al Servicio de nuestros alumnos y del país. Del mismo modo,

\section{REFERENCIAS}

1. Archivo del Arzobispado de Santiago. Decretos del Arzobispado de Santiago, 1924-1929, Libro 28.

2. El Mercurio, 27 de marzo de 1930, 3.

3. Vargas JE, Chuaqui B, Duarte I. Médicos de Ciencia y Conciencia. La Escuela de Medicina de la Pontificia Universidad Católica de Chile. Capítulo 3, Marzo 2005. debemos enfatizar que nuestra Escuela de Medicina está inserta y pertenece a una Pontificia Universidad Católica, lo que nos impone un desafío y una responsabilidad aún mayor, junto a la formación de excelencia, y en palabras de nuestro fundador Monseñor Carlos Casanueva, estamos llamados a formar médicos no solo de ciencia, sino también de conciencia, al servicio de la sociedad.

Para finalizar, creemos importante citar al Rector, Dr. Pedro Pablo Rosso, en su discurso de inauguración del año académico del año 1996, en donde destacaba que «debemos construir una Escuela de Medicina arraigada en la fe cristiana, comprometida vitalmente con la labor universitaria; agente de progreso de la Medicina; instrumento eficaz para la evangelización de la cultura sanitaria. Con una comunidad universitaria auténticamente humana, integrada por personas que buscan sinceramente la verdad, animada por un espíritu de libertad y de caridad, caracterizada por el diálogo sincero y el respeto a los derechos de cada uno». Con estos objetivos debemos trabajar arduamente en esta comunidad académica.

4. Vargas JE, Chuaqui B, Duarte I. Médicos de Ciencia y Conciencia. La Escuela de Medicina de la Pontificia Universidad Católica de Chile. Capítulo 8, Marzo 2005.

5. www.escuela.med.puc.cl

6. Vargas JE, Chuaqui B, Duarte I. Médicos de Ciencia y Conciencia. La Escuela de Medicina de la Pontificia Universidad Católica de Chile. Capítulo 4, Marzo 2005.

7. Revista Educación Médica UC, № 14, 1996. 